\title{
The weight of truth: \\ Lessons for minimalists from Russell's Gray's Elegy argument
}

\author{
Tim Button \\ button@cantab.net
}

This is a preprint of a Paper accepted for publication in the Proceedings of the Aristotelian Society (C) 2014.

Truth can seem mysterious. Paul Horwich's minimalism claims to dissolve all appearance of mystery, telling us that the concept of truth is exhausted by a single scheme:

MT. The proposition that $p$ is true iff $p$

Nothing, it seems, could be simpler. Unfortunately, there are subtle difficulties with treating MT as a scheme, in anything like the ordinary sense. In the end, these difficulties lead to the demise of minimalism about truth.

Just as there are minimalists about truth, so there are minimalists about reference and satisfaction. Where minimalists about truth focus on propositions, minimalists about reference and satisfaction focus on propositional constituents. But minimalists of all stripes encounter very similar problems. Indeed, their difficulties arise from foundational issues concerning propositions and propositional constituents. These points can be traced back to Bertrand Russell's Gray's Elegy argument.

I therefore begin my case against minimalism by introducing the idea of a propositional constituent and extracting some lessons from the Gray's Elegy argument (SI). I then introduce minimalism about reference (\$II), and show how it is undermined by the Gray's-Elegy-inspired lessons ( $\$ I I I)$. The argument against minimalism about reference is easy to translate into an argument against minimalism about truth and satisfaction ( $\$ I V)$. Moreover, this argument helps us to understand one of Donald Davidson's arguments against minimalism $(\$ \mathrm{~V})$. I close by rejecting three possible responses which the minimalist might try to make ( $\$ S$ VI-VIII), and by attempting to demotivate minimalism ( $($ IX).

\section{Three lessons from Russell's Gray's Elegy argument}

In this paper, I raise problems for minimalists via foundational considerations which can be traced back to Russell's Gray's Elegy argument. This section aims to introduce these foundational considerations. I start by briefly introducing the idea of a propositional constituent. I then present the Gray's Elegy argument. And I close the section by drawing three lessons which will be relevant for my subsequent discussion of minimalism. 
Propositions and their constituents. Suppose we think of propositions as in some way composed out of various entities. For example, we might think that the proposition that Hesperus rotates has a constituent which denotes, refers to, or picks out Hesperus. It is customary to indicate this constituent thus: 〈Hesperus〉. In general, then, angled brackets are supposed to introduce us to propositional constituents. In the simplest case, where an entire sentence is enclosed between angled brackets, we are introduced to a fully fledged proposition. So, deploying our new notation, we can say that $\langle$ Hesperus〉 is a constituent of $\langle$ Hesperus rotates $>$ (i.e. of the proposition that Hesperus rotates).

This use of angled brackets is entirely ubiquitous among philosophers discussing minimalism. Marian David (2008, p. 287) suggests a helpful way to think about their intended use: just as quotation marks typically indicate semantic ascent, so angled brackets are supposed to indicate intensional ascent. Of course, one might have many questions about intensional ascent - indeed, the central message of this paper is that the use of angled brackets has led minimalists astray - but for now, let us proceed.

We have said that $\langle$ Hesperus $\rangle$ is to be a constituent of $\langle$ Hesperus rotates $\rangle$. But we now face a crucial (and familiar) decision-point, concerning how we should think of propositions and their constituents. Astronomical observations tell us that Hesperus = Phosphorus; but does $\langle$ Hesperus $\rangle=\langle$ Phosphorus $\rangle$, and does $\langle$ Hesperus rotates $\rangle=\langle$ Phosphorus rotates $\rangle$ ?

It is easy to motivate a negative answer to these questions. No observations were needed to determine the truth of $\langle$ Hesperus $=$ Hesperus $\rangle$, but it required serious effort to show that $\langle$ Hesperus $=$ Phosphorus $\rangle$ was true. As such, we might well want to say that these are different propositions. And since they differ only in that one contains $\langle$ Hesperus $\rangle$ where the other contains $\langle$ Phosphorus $\rangle$, we shall also say that $\langle$ Hesperus $\rangle \neq\langle$ Phosphorus $\rangle$. For similar reasons, we shall say that $\langle$ Hesperus rotates $\rangle \neq\langle$ Phosphorus rotates $\rangle$.

Call this the broadly Fregean approach to propositions and propositional constituents. It is only broadly Fregean, since you can follow this approach whilst disagreeing with Frege's detailed account of sense. What the approach preserves is just Frege's claim, that $\langle$ Hesperus rotates $\rangle \neq\langle$ Phosphorus rotates $\rangle$ and that $\langle$ Hesperus $\rangle \neq\langle$ Phosphorus $\rangle$.

Summarising the Gray's Elegy argument. It is at this point that Russell's Gray's Elegy argument gets going (1905, pp. 485-7). I shall outline the argument, following Michael Potter's (2000, pp. 124-5) reconstruction very closely.

One might, naively, think that angled brackets indicated a one-place function, so that, in general, if $a=b$ then $\langle a\rangle=\langle b\rangle$. (For comparison, consider how we use curly brackets in set theory.) However, given a broadly Fregean approach to propositional constituents, this is mistaken: 〈Hesperus〉 $\neq\langle$ Phosphorus $\rangle$ even though Hesperus $=$ Phosphorus. Potter summarises this point as follows:

The notation ' $\langle c\rangle$ ' is misleading: $\langle c\rangle$ does not depend functionally on $c$. (Potter 2000, p. 124). ${ }^{1}$

1 Potter has 'it' after the semicolon, where I have ' $\langle c\rangle$ '. Potter also uses a capital ' $C$ ' where I use ' $c$ '; I have silently adjusted this, and all subsequent quotations, from Potter. 
Evidently it will not do to employ misleading notation. But, we should not be too quick to do away with angled brackets. Recall that we wanted to say that there is some relationship between 〈Hesperus〉 and Hesperus: the former refers to, or denotes, or picks out the latter. And we shall want to generalise this thought, offering something like the scheme: $\langle c\rangle$ denotes c. Unfortunately, the generality of this scheme requires the use of angled brackets (or some similar notational expedient). As Potter points out:

If we use a new symbol [in place of ' $\langle c\rangle$ '] , say ' $d$ ', then we have to express the relationship we want by saying that $d$ denotes $c$. This is no longer in any way explanatory of the general relationship which we wanted to describe, but has to be expressed afresh for each denoting concept $d$. (Potter 2000, p. 124)

We seem, then, to be forced to use angled brackets; but we must find a way to avoid being misled by them. Potter continues:

The most natural way for us to designate $\langle c\rangle$, of course, is as the meaning of ' $c$ ', but 'the meaning of " $c$ "' is not a function of $c$ any more than $\langle c\rangle$ is: it is rather a function of the phrase ' $c$ ', so if we try to express what we want by saying that the meaning of ' $c$ ' denotes $c$, we are making the relationship between meaning and denotation 'linguistic through the phrase'. (Potter 2000, pp. 124-5; Potter's emphasis $)^{2}$

At this point, Russell insisted that 'the relationship of meaning and denotation is not merely linguistic through the phrase: there must be a logical relation involved' (1905, p. 486). This is the Gray's Elegy argument, against a broadly Fregean approach to propositional constituents.

For his own part, of course, Russell is associated with a position according to which Hesperus itself is the propositional constituent in the proposition 〈Hesperus rotates $\rangle$. Indeed, on a broadly Russellian approach to propositional constituents, we would be led to say that $\langle$ Hesperus $\rangle=$ Hesperus $=$ Phosphorus $=\langle$ Phosphorus $\rangle$. At this point, the use of angled brackets would cease to be misleading - after all $\langle c\rangle$ now trivially depends functionally on $c$ — but they would be entirely redundant.

Three lessons from the Gray's Elegy argument. One might think that the Gray's Elegy argument undermines any broadly Fregean approach to propositional constituents. I make no such claim. I have rehearsed the Gray's Elegy argument, only because I think that it provides three important lessons for anyone who embraces a broadly Fregean approach. These lessons are as follows:

2 Note, too, that the meaning of ' $c$ ' is not a function of $c$; and, indeed, that ' $c$ ' is not a function of $c$. This latter point forms the locus of Read's (1997) discussion of a puzzle due to Reach (1938) and Anscombe (1957). 
Lesson 1 . The use of angled brackets is potentially misleading, since $\langle c\rangle$ is not a function of $c$.

Lesson 2. However, abandoning the use of angled brackets altogether would leave us unable to say anything general about reference.

Lesson 3. The best explanation of the use of angled brackets makes intensional ascent depend upon semantic ascent, as follows:

$$
\langle\ldots\rangle={ }_{\mathrm{df}} \text { the meaning of '...' (in this language) }
$$

Of course, this does not alter the fact that $\langle c\rangle$ is not a function of $c$.

I shall invoke these three lessons several times in what follows, to argue against minimalism about reference, truth, and satisfaction, in that order.

\section{Minimalism about reference, and the challenge of comprehensiveness}

The preceding discussion is immediately relevant to the first minimalist position that I shall consider: Horwich's minimalism about reference.

Minimalists about reference think that propositional constituents are the primary referring entities. Moreover, minimalists think that it is relatively easy to know everything there is to know about reference. In particular, they believe that everything there is to know about reference is exhausted by the following scheme (Horwich 1998, pp. 116, 130):

MR. $\forall x(\langle c\rangle$ refers to $x$ iff $c=x)$

(This is obviously in the same ballpark as the scheme ' $\langle c\rangle$ refers to $c$ ', mentioned in SI. However, it is a slight improvement, since it allows for the possibility of reference failure, as when we substitute 'Pegasus' for ' $c$ '.)

By rolling up everything into a single scheme, the minimalist seeks to demystify reference. There is, however, an immediate difficulty with the thought that MR exhausts everything there is to know about reference. There are propositional constituents which cannot be expressed in this language. (To take a simple example: this language does not contain a name for each and every real number.) Consequently, there are propositional constituents whose reference condition cannot be specified by any instance (in this language) of $M R .{ }^{3}$ So the theory of reference which consists of all the instances (in this language) of MR is not comprehensive, in the sense that it does not provide us with a reference condition for every propositional constituent that (putatively) refers.

3 A reference condition is a statement of what (if anything) a (putatively) referring entity refers to. (Compare the notion of a reference condition with the the notion of a proposition's truth condition.) A reference condition is sometimes called a reference, but then we must distinguish between the reference and the referent (the denoted object), which is apt to confuse. 
The minimalist's theory of reference must, though, be comprehensive. After all, if there is some propositional constituent which refers, but whose reference condition the minimalist cannot specify, then clearly the minimalist has not told us everything there is to know about reference. (I discuss a further reason for the minimalist to require comprehensiveness in \$VIII.)

The minimalist therefore faces a challenge: she must find a way to provide a comprehensive theory, without giving up on MR. For the bulk of this paper, I shall explore one response to this challenge (I consider an alternative response in \$VII). The response I shall consider is Horwich's own preferred response to the challenge of comprehensiveness, and it is inadequate. (More precisely, in fact, this is Horwich's (1998, pp. 17-20) preferred response in the case of minimalism about truth. However, it would be remarkable if he were to treat the case of reference differently, and it is easy to translate his response across.)

Aware of the challenge of comprehensiveness, Horwich denies that MR should be treated as an axiom scheme in the ordinary sense. Rather, Horwich maintains that MR illustrates a certain general structure that propositions can have. That propositional structure is a 'single one-place function' (1998, p. 19n3), which we can illustrate even more clearly as:

$$
\langle\forall x(\langle c\rangle \text { refers to } x \text { iff } c=x)\rangle
$$

The minimalist's theory of reference is then generated as follows: its axioms are exactly those propositions that result from inputting each and every (putatively referring) propositional constituent into this one-place function. Thus, if one inputs $\langle$ Hesperus $\rangle$ into this function, one obtains $\langle\forall x(\langle$ Hesperus $\rangle$ refers to $x$ iff Hesperus $=x)\rangle$. Crucially, though, the input to this function can be any propositional constituent, rather than just those which are expressible in this language.

In brief, then, the minimalist about reference claims to answer the challenge of comprehensiveness by appealing to a 'one-place function (the propositional structure)' which can be applied to any propositional constituent (Horwich 1998, p. 19n3). In what follows, I show that this is untenable.

\section{Applying the lessons from the Gray's Elegy argument}

So far, the minimalist has not yet declared in favour of either a broadly Fregean or Russellian approach to propositional constituents. Horwich himself explicitly accepts the existence of both kinds of propositional constituents (1998, pp. 90-2). Indeed, he claims that minimalism will be the correct theory of truth for either kind of proposition (1998, p. 17).

I return to this claim in $\$ I V$ and $\$ V I$. For now, it suffices to note that minimalism cannot be the correct theory of reference for Russellian propositional constituents. As we saw in \$I, on a Russellian approach to propositional constituents, angled brackets are redundant. Accordingly, the Russellian approach would have us read MR as: 


$$
\mathrm{MR}_{1} . \forall x(c \text { refers to } x \text { iff } c=x)
$$

This tells us, absurdly, that objects always and only refer to themselves.

Of course, the Russellian approach is not the only alternative to a broadly Fregean approach. An alternative would be a position according to which $\langle$ Hesperus $\rangle \neq$ Hesperus, but $\langle$ Hesperus $\rangle=\langle$ Phosphorus $\rangle$. More generally, on this approach, angled brackets would represent a function according to which $\langle a\rangle=\langle b\rangle$ iff $a=b$. On such an approach to propositional constituents, however, it will evidently be easy to eliminate any use of the predicate 'refers'. And so this approach to propositional constituents will lead, not to minimalism about reference, but to a redundancy theory of reference.

Consequently, minimalists about reference must adopt a broadly Fregean approach to propositional constituents. And, as such, the three lessons from the Gray's Elegy argument apply.

Concerning Lesson 1. Given a broadly Fregean approach to propositional constituents, $\langle c\rangle$ is not a function of $c$. Consequently, the use of angled brackets in MR is potentially misleading. This is just Lesson 1 of the Gray's Elegy argument.

The potential to mislead is not, unfortunately, just an abstract possibility. In $\$ I I$, our minimalist responded to the challenge of comprehensiveness by claiming that MR indicates a particular propositional structure - a 'single one-place function' - which takes as inputs any propositional constituent (including those we cannot express). However, MR would indicate a one-place function if, and only if, angled brackets indicated a one-place function. We have just seen that they do not. The minimalist, then, has been misled by her own notational devices.

(Actually, the argument which I have just given involves a very slight oversimplification. Here is the unsimplified argument. Suppose the minimalist theory is to consist of Russellian propositions which provide the reference conditions for Fregean propositional constituents. Then the minimal theory should contain the Russellian proposition that $\forall x(\langle$ Hesperus $\rangle$ refers to $x$ iff Hesperus $=x$ ) (with angled brackets still indicating Fregean propositional constituents). Since this is a Russellian proposition, it has Hesperus as one constituent, and 〈Hesperus〉 as another. And, since there is no 'backward road' (Russell 1905, p. 487) from Hesperus to 〈Hesperus〉, the problem is as stated above. But suppose, instead, that the minimalist theory is to consist of Fregean propositions which provide the reference conditions for Fregean propositional constituents. Then the proposition in question will have $\langle$ Hesperus $\rangle$ as one constituent, and $\langle\langle$ Hesperus $\rangle\rangle$ as another. Nevertheless, there is still no 'backward road' from $\langle$ Hesperus $\rangle$ to $\langle\langle$ Hesperus $\rangle\rangle$ : it is illuminating to discover that $\langle$ Hesperus $\rangle$ is Tim's favourite propositional constituent just as it is illuminating to discover that Hesperus is Phosphorus - so that $\langle\langle$ Hesperus $\rangle\rangle \neq$ $\langle$ Tim's favourite propositional constituent $\rangle$. So MR still cannot indicate a one-place function.) 
of ' $\langle c\rangle$ ' in MR, which does not suggest a functional dependence on $c$. For example, we might use some primitive new symbol ' $d$ '. We would then have to rewrite MR as follows:

$$
\mathrm{MR}_{2} . \forall x(d \text { refers to } x \text { iff } c=x)
$$

This scheme illustrates a two-place function, with gaps marked by ' $d$ ' and ' $c$ '. However, absurdity follows very quickly indeed, if we are allowed to input absolutely any pairs of propositional constituents into this function. Accordingly, the minimalist must impose some restrictions on which pairs of inputs are permissible.

What the minimalist will want to say, of course, is that the only permissible pairs of inputs are such that the first refers to the second. However, if the minimalist offers this as an explicit constraint in specifying her minimal theory of reference, then she will have invoked precisely the concept that she was trying to deflate away, undercutting her own aims.

The minimalist might try to maintain that her theory of reference consists of all correct instances of the two-place function illustrated by $\mathrm{MR}_{2}$, whilst adding that nothing more can be said about which instances are correct. This, however, is to give up on minimalism. If there is nothing in common between the propositions in the minimal theory of truth beyond their inexplicable correctness, then we will have given up on any hope of systematically specifying any general relationship of reference.

In short, since the minimalist wants to keep the general relationship of reference in view, she must rely upon angled brackets in formulating her theory. This is just Lesson 2 from the Gray's Elegy argument.

Concerning Lesson 3. Since the minimalist must employ angled brackets, we are still owed an explanation of their meaning. And the most natural thought is as follows: propositional constituents are first introduced to us just as what certain phrases express. Consequently, the most natural explanation of angled brackets will be 'linguistic through the phrase'; it will make intensional ascent depend upon semantic ascent. Indeed, Horwich himself explicitly makes intensional ascent depend upon semantic ascent, declaring:

I am employing the convention that surrounding any expression, $e$, with angled brackets, '〈' and '〉', produces an expression referring to the propositional constituent expressed by e. (Horwich 1998, p. 18n3; see also his 2009b, p. 87n8.)

Otherwise put, Horwich offers the following definition:

$$
\langle\ldots\rangle={ }_{\mathrm{df}} \text { the propositional constituent expressed by '...' (in this language) }
$$

This is just Lesson 3 from the Gray's Elegy argument.

The demise of minimalism. Given Lesson 3, we should rewrite MR as follows: 
$\mathrm{MR}_{L} . \forall x$ (the propositional constituent expressed by ' $c$ ' (in this language) refers to $x$ iff $c$ $=x)$

But, with MR thus unpacked, we must now revisit the minimalist's answer to the challenge of comprehensiveness from $\$ I I$. The minimalist held that we should not treat $\mathrm{MR}_{L}$ (i.e. $M R$ ) as an axiom scheme in the conventional sense. Rather, we should treat $\mathrm{MR}_{L}$ as illustrating a one-place function. The axioms of the minimalist's theory of reference are then exactly those propositions that result from inputting each and every (putatively referring) propositional constituent into this one-place function.

Unfortunately for the minimalist, $\mathrm{MR}_{L}$ does not point to a one-place function, any more than does Mr. This is immediate from the fact that the propositional constituent expressed by ' $c$ ' (in this language) is not a function of $c$.

(As before, this is a very slight oversimplification. Here is the unsimplified version. If the minimalist theory consists of Russellian propositions which provide reference conditions for Fregean propositional constituents, then the problem is as just stated. If the minimalist theory consists of Fregean propositions which provide reference conditions for Fregean propositional constituents, then the problem is just that there is no backward road from $\langle$ Hesperus〉 to 〈the (Fregean) propositional constituent expressed by 'Hesperus' (in this language $)\rangle$.)

At this point, I expect that the minimalist will ask us to allow her a grain of salt in her attempt to specify her theory of reference. Surely, she might protest, I understand what $M R_{L}$ is getting at?

When it comes to instances of $\mathrm{MR}_{L}$ in this language, I am happy to grant the grain of salt. I am happy to concede that $\mathrm{MR}_{L}$ successfully specifies a reference condition for every propositional constituents which is expressible in this language. After all, the reference condition for $\langle c\rangle$ is given to me with a clause which is linguistic through the phrase ' $c$ ' in this language, and I am happy to concede that all such clauses are intelligible. Crucially, though, $\mathrm{MR}_{L}$ leaves me entirely at sea, when it comes to propositional constituents are inexpressible in this language to me (at least in principle). After all, whenever this language lacks the means to express a constituent, that constituent's reference condition can hardly be specified by mentioning some phrase which, when used in this language, expresses that constituent! Consequently, $\mathrm{MR}_{L}$ cannot even begin to gesture at a reference condition for propositions which cannot be expressed in this language. The challenge of comprehensiveness is completely unanswered.

Here, then, is the problem for minimalists in a nutshell. The minimalist position relies upon intensional ascent, which is conventionally symbolised with angled brackets. Since minimalists must be broadly Fregean about propositional constituents, they must explain intensional ascent in terms of semantic ascent in this language. But in that case, they cannot address the challenge of comprehensiveness. 


\section{IV.Minimalism about truth}

This concludes my case against minimalism about reference. I now want to show that essentially the same problem arises for minimalism about truth. This should be no surprise, given the intimate connection between truth and reference. However, it is worth spelling out the problem in a little detail.

The challenge of comprehensiveness. Horwich's minimalism about truth involves the scheme (e.g. 1998, pp. 17-20):

$$
\text { MT. }\langle p\rangle \text { is true iff } p
$$

The minimalist's theory of truth must, though, be comprehensive, in the sense that it must provide a truth condition for each truth bearer. And this a serious challenge, since certain truths cannot be expressed in this language.

As such, and as in $\$ I I$, the minimalist maintains that MT indicates a propositional structure: a single one-place function that can be applied to every proposition to generate its truth condition. Since the propositions that we input into this propositional structure are not limited to those which are expressible in this language, the minimalist thereby claims to have answered the challenge of comprehensiveness.

Which approach to propositions? The minimalist must now consider how she should conceive of propositions.

We saw in $\ I I I$ that a Russellian approach to propositional constituents immediately yields a patently absurd theory of reference. A Russellian approach to propositions will not yield a patently absurd theory of truth (at least, not obviously). However, the theory of truth will not look particularly minimalist. After all, to be on the road to a correspondence theory (or perhaps an identity theory) of truth, very little more is required than that Hesperus is a component of $\langle$ Hesperus rotates $\rangle$. Hartry Field has stated this problem very nicely:

Russell viewed atomic propositions as complexes consisting of an $n$-place relation and $n$ objects, in some definite order. But an account of truth for such propositions is obvious: Such a proposition is true iff the objects taken in that order stand in the relation. It can hardly be a matter of philosophical controversy whether this definition of truth is correct, given the notion of proposition in question, so what is there for the minimalist and the full-blooded correspondence theorist to disagree about? (Field 1992, p. 323)

This gives a prima facie reason for the minimalist to adopt the Fregean account of propositions. It is, though, only a prima facie reason. After all, there may be alternative approaches to propositions which are not broadly Fregean, but which equally avoid thinking 
that $\langle$ Hesperus rotates $\rangle$ consists of the ordered pair of Rotation and Hesperus. ${ }^{4}$ I shall revisit this matter in $\$$ VI. For now, I shall take it that the minimalist about truth should focus on Fregean propositions. Consequently, we can apply the lessons from the Gray's Elegy Argument.

Concerning Lesson 1. Given a broadly Fregean approach to propositions, 〈Hesperus rotates〉 is distinct from $\langle$ Phosphorus rotates $\rangle$. As such, neither proposition depends functionally upon the rotation of Hesperus (i.e. Phosphorus), and so the use of angled brackets - or, equivalently, unreflective use of the phrase 'the proposition that...' - is potentially misleading. Indeed, it has already misled the minimalist. Her answer to the challenge of comprehensiveness involved the claim that MT indicated a one-place function. But it could do this if, and only if, angled brackets marked some function.

Concerning Lesson 2. To avoid being misled, we might consider a two place function:

$$
\mathrm{MT}_{2} \cdot q \text { is true iff } p
$$

However, the inputs to this function would need to be somehow constrained. Reflecting upon this point, it is clear that the minimalist must use angled brackets, if she wants to retain any hope of systematically specifying some general conception of truth.

Concerning Lesson 3. The minimalist still owes us an explanation of these angled brackets. The natural thing to do, of course, is just to explain intensional ascent via semantic ascent. And this is exactly what Horwich suggests (see above).

The demise of minimalism. Given how we are to read angled brackets, scheme MT becomes:

$$
\mathrm{MT}_{L} \text {. the proposition expressed by ' } p \text { ' (in this language) is true iff } p
$$

Of course, this cannot indicate a one-place function. More generally, since it involves semantic ascent in this language, it cannot help us concerning truth conditions for propositions which cannot be expressed in this language. Thus, minimalism about truth fails to address the challenge of comprehensiveness.

Minimalism about satisfaction. The argument against minimalism about truth is concluded. Before moving on, though, it is worth broadening the argument. Minimalists treat truth as a property of propositions; propositions are expressed by sentences; and sentences are just zero-place predicates. So minimalism about truth is just minimalism about zero-place satisfaction. This observation suggests that we might consider minimalism about satisfaction more generally.

4 Though Field (1992, pp. 322-3) also points out that regarding propositions as sets of possible worlds is unlikely to yield a distinctively minimalist thesis. 
The next simplest case to consider is minimalism about satisfaction of one-place predicate-like propositional constituents. To just this end, Horwich (1998, pp. 116, 130) suggests the following scheme:

MS. $\forall x(x$ satisfies $\langle F\rangle$ iff $F x)$

Similar schemes could be given to deal with satisfaction of $n$-place predicate-like propositional constituents (for arbitrary $n$ ). But it is obvious that all such proposals will face the same problems as faced by the minimalist about truth: they cannot address the challenge of comprehensiveness.

\section{Davidson on minimalism}

I have constructed my argument against minimalism around three lessons which I extracted from Russell's Gray's Elegy argument. However, I could equally well have built my argument around one of Davidson's objections against minimalism. That objection is fascinating, but highly compressed; indeed, it is sufficiently compressed for me to want to quote it in full:

How are we to understand phrases like 'the proposition that Socrates is wise'? In giving a standard account of the semantics of the sentence 'Socrates is wise', we make use of what the name 'Socrates' names, and of the entities of which the predicate 'is wise' is true. But how can we use these semantic features of the sentence 'Socrates is wise' to yield the reference of 'the proposition that Socrates is wise'? Horwich does not give us any guidance here. Could we say that expressions like 'the proposition that Socrates is wise' are semantically unstructured, or at least that after the words 'the proposition that' (taken as a functional expression) a sentence becomes a semantically unstructured name of the proposition it expresses? Taking this course would leave us with an infinite primitive vocabulary, and the appearance of the words 'Socrates is wise' in two places in the schema would be of no help in understanding the schema or its instances. A further proposal might be to modify our instance of the schema to read:

The proposition expressed by the sentence 'Socrates is wise' is true if and only if Socrates is wise.

But following this idea would require relativizing the quoted sentence to a language, a need that Horwich must circumvent.

So let me put my objection briefly as follows: the same sentence appears twice in instances of Horwich's schema, once after the words 'the proposition that', in a 
context that requires the result to be a singular term, the subject of a predicate, and once as an ordinary sentence. We cannot eliminate this iteration of the same sentence without destroying all appearance of a theory. But we cannot understand the result of the iteration unless we can see how to make use of the same semantic features of the repeated sentence in both of its appearances-make use of them in giving the semantics of the schema instances. I do not see how this can be done. (Davidson 1996, pp. 273-4)

Horwich has published two replies to Davidson's objection (2001, pp. 153-4; 2009a, pp. 53-6; see also his 1998, p. 133). Whilst these responses differ slightly, both assume that Davidson's challenge merely evinces 'a squeamishness about Fregean "that"-clauses' (2009a, p. 55). More precisely, Horwich thinks that Davidson has simply raised the following worry:

'That'-clauses cannot be regarded as referring expressions, because there is no way of seeing how their referents would be determined by the referents of their component words. (Horwich 2009a, p. 53; see also 2001, pp. 152-3; 2009c, p. $76 n 20)$

As a result, Horwich's main line of response against Davidson's objection is simply to explain how intensional ascent works. Naturally, he glosses it in terms of semantic ascent (in this language).

I think that Horwich has misunderstood Davidson's objection. A page before the lengthy quotation just offered, Davidson has praised Horwich, on the grounds that Horwich seems 'to have accepted the challenge other deflationists have evaded, that of saying something more about an unrelativized concept of truth than we can learn from Tarski's definitions' (1996, p. 272). But, in the lengthy quoted passage, Davidson maintains that Horwich will end up with a relativized concept of truth after all, if intensional ascent is articulated in terms of semantic ascent. None of this discussion of relativization has anything, though, to do with a general 'squeamishness about Fregean "that"-clauses'.

We reach a much better understanding of Davidson's objection, if we read him as advancing something close to the argument I presented in \$IV. Indeed, Davidson's objection makes use of all three of the lessons that I extracted from the Gray's Elegy argument.

Concerning Lesson 1. Davidson starts by worrying that there is no direct route to $\langle$ Socrates is wise > from Socrates ('what the name "Socrates" names') and Wisdom (or rather, 'the entities of which the predicate "is wise" is true').

Although Davidson does not make this explicit, the worry is really only made serious given a broadly Fregean approach to propositions. Indeed, on the Russellian approach to propositions, 〈Socrates is wise $\rangle$ is just the ordered pair consisting of Wisdom and Socrates, so that there is a direct and obvious route from Wisdom and Socrates to 〈Socrates is wise $\rangle$. By contrast, on a broadly Fregean approach to propositions, 〈Socrates is wise〉 is not a function of Socrates's wisdom. 
Concerning Lesson 2. Davidson then considers treating 'the proposition that Socrates is wise' as semantically unstructured. But if such expressions are semantically unstructured, then we would do just as well to have referred to them using primitive symbols with no apparent complexity. At this point, we would have introduced 'an infinite primitive vocabulary', thereby 'destroying all appearance of a theory'. This is exactly on a par with Potter's point, that the result is no longer in any way explanatory of the general relationship which we wanted to describe, but has to be expressed afresh for each' proposition (2000, p. 124).

Concerning Lesson 3. Davidson entertains a 'further proposal', which explains intensional ascent in terms of semantic ascent. Accommodating the point that this needs to be relativized to a language, the 'further proposal' essentially comes to the following:

$$
\langle\ldots\rangle={ }_{\mathrm{df}} \text { the proposition expressed by '...' (in this language) }
$$

Davidson's argument against minimalism consequently runs through all three of the lessons from the Gray's Elegy argument. Thus far, then, Davidson and I agree completely in our objections against minimalism. However, we part company over why the minimalist cannot explain intensional ascent in terms of semantic ascent in this language.

Davidson does not explicitly state his reasons at this point, but we can reconstruct them easily enough. Earlier in the paper under discussion (and elsewhere) Davidson argues against theories of truth which involve explicit relativizations to languages as follows. Since they involve relativizations to languages, each theory defines only a relativized truth predicate. However, the theories themselves provide no account of what all of these different predicates 'have in common' (Davidson 1990, pp. 285, 288, 295; also 1996, p. 269). And this is unacceptable, not least because we are given no 'idea how to apply the concept [of truth] to a new case, whether the new case is a new language or a word newly added to a language' (1990, p. 287). Almost certainly, Davidson thinks that the same problem will affect the minimalist who seeks to make intensional ascent depend upon semantic ascent in this language.

I am not sure whether Davidson is right about this point, but I shall not explore this matter here. For present purposes, it suffices to note that Davidson's ultimate objection against minimalism is somewhat different from my own. At the risk of repetition: my objection is that, if intensional ascent depends upon semantic ascent in this language, then the minimalist cannot address the challenge of comprehensiveness.

Nonetheless, Lessons 1-3 constitute a shared core between Davidson's argument and my own. Moreover, this core is also shared with Russell's Gray's Elegy argument. We all agree that, having adopted a broadly Fregean approach to propositions or propositional constituents, intensional ascent must be explained via semantic ascent in this language; and we all agree that this is problematic; but we part company over why it is problematic. 


\section{A purely functional view of propositions and the redundancy theory of truth}

My central argument against minimalism is concluded. Over the next three sections, I shall consider some possible responses from minimalists, and explain why they are lacking. In all three cases, I shall focus exclusively on the case of truth, but similar comments will apply to the cases of reference and satisfaction.

A central contention of the paper, so far, is that the various minimalist schemes (MR, MT, and MS) cannot indicate a one-place function. In this section, I want to offer a related objection: if these schemes did indicate a one-place function, then we would not be pushed towards minimalism, but towards the redundancy theory.

Suppose that the minimalist simply insists that MT indicates a one-place function. In so doing, she must reject a broadly Fregean approach to propositions (at least, insofar as she wants to be a minimalist about the truth of such things). There may be some leeway, concerning which approach to propositions she might adopt. For concreteness, though, I shall suppose that our minimalist attempts show that MT indicates a genuinely one-place function, by showing how to define it by composition from smaller functions. The relevant functions are as follows:

$T(x)$ : a function which takes a proposition $x$ as input, and outputs a new proposition by predicating truth of $x$.

$B(x, y)$ : a function which takes two propositions $x$ and $y$ as inputs, and outputs a new proposition by biconditionalising them, with $x$ as the left- and $y$ as the right-hand side (as it were).

The minimalist may now maintain that MT offers a linguistic gloss on the (genuinely) oneplace composite function, $B(T(x), x)$. Call this approach to propositions, the purely functional approach. ${ }^{5}$ (This approach is, of course, compatible with the Russellian view that atomic propositions are complexes consisting of an $n$-place relation and $n$ objects.)

My claim is as follows: if (if) there are propositions of a purely functional sort, then the redundancy theory of truth is preferable to minimalism when it comes to such propositions. To establish this claim, I shall show why the purely functional approach to propositions overcomes a famous (and supposedly decisive) objection to the redundancy theory.

The redundancy theory of truth holds that all truth-predications are eliminable. However, it famously runs into difficulties when confronted with claims like:

(1) everything the Pope asserts is true

5 This approach was urged upon me (independently) by George Bealer, Ted Sider and Mark Jago. Many thanks to them all for discussions and correspondence on this point. Horwich indicates something like this, with his talk of 'compositional structure' (1998, p. 91) and 'form' (2009, pp. 43-5). Moreover, if the minimalist is prepared to countenance that propositions have constituents, then they seem already to have adopted (some version of) the purely functional approach. 
This might naturally be read as follows:

(2) $\forall x$ (if the Pope asserts $x$, then $x$ is true)

Now, if truth were redundant, we would expect to be able to eliminate the truth predicate from this sentence, offering:

(3) $\forall x$ (if the Pope asserts $x$, then $x$ )

Unfortunately, this would have ill-formed instances, such as:

(4) if the Pope asserts Tarski's favourite proposition, then Tarski's favourite proposition.

This is generally taken to be a knockdown objection to the redundancy theory of truth. Indeed, minimalism takes itself to preserve the spirit of the redundancy theory, whilst recognising that the redundancy theory itself must be abandoned (see e.g. Horwich 1998, pp. 38-40).

However, the purely functional approach to propositions has no particular difficulty in dealing with the proposition expressed by (1). To handle it, we first need to help ourselves to two more functions, namely:

$P(x)$ : a function which takes a proposition $x$ as input, and outputs a new proposition by predicating papal assertion of $x$.

$C(x, y)$ : a function which takes two propositions $x$ and $y$ as inputs, and outputs a new proposition by conditionalising them, with $x$ as the antecedent and $y$ as the consequent (as it were).

If $T$ and $B$ are acceptable functions, then $P$ and $C$ are too. Finally, we need some operation which applies to functions, such as:

$\mathrm{A}_{x} \varphi(x)$ : when $\varphi$ is a one-place function which takes an input $x$ and outputs a proposition by predicating $F$ of $x$, this operation takes $\varphi$ as an input and outputs a proposition by predicating $F$ of everything.

Some such operation is required by an advocate of the purely functional account of propositions, in order to allow her to deal with those propositions which we express with universal quantifiers.

Now, $C(P(x), x)$ is a one-place function from propositions to propositions. One might worry that, if $x$ is Tarski's favourite proposition, then $C(P(x), x)$ leads to the ill-formed (4). But an advocate of the purely functional approach to propositions is already committed to claiming that this is a mistake; for $B(T(x), x)$ had better not lead to the ill-formed 'Tarski's 
favourite proposition is true iff Tarski's favourite proposition', in the same case. Accordingly, since $C(P(x), x)$ is a one-place function, $\mathrm{A}_{x} C(P(x), x)$ is a bona fide proposition. Indeed, a moment's reflection indicates that it is just the proposition expressed by (1). But notice that this proposition does not involve any predication of truth. Hence, on the purely functional approach to propositions, we can handle the proposition expressed by (1) without invoking truth. Truth is redundant after all!

We are now in a strange situation. On the one hand, there is a supposedly knockdown argument against the eliminability of truth. On the other, the purely functional approach to propositions (if legitimate) allows us to eliminate truth completely.

In fact, there is no serious tension here. The original argument shows us that the truthpredicate cannot be eliminated from our language without expressive loss. However, the purely functional approach to propositions (if legitimate) allows us to eliminate truth from the propositions we express with our language. This mismatch between propositions and the language we use to express them is, in turn, readily explicable. The purely functional approach to propositions countenances no fundamental distinction between predication and logic operations: both are functions which invariably output propositions. Consequently, the purely functional approach to propositions can see no real distinction between the truth predicate and the truth operator. ${ }^{6}$ However, the truth operator, unlike the truth predicate, is provably redundant. Ultimately, then, it is no surprise that the purely functional approach to truth leads us away from minimalism, towards a redundancy theory of truth (for propositions).

But all of this is just more salt in the minimalist's wounds. The minimalist wanted to maintain that MT indicates a one-place function into which we can plug any proposition, and that this one-place function allows us to deal with all predications of truth. The purely functional approach to propositions would enable us to make sense of this idea; but it would equally show that there is no need to get involved with truth predications (at the level of propositions) in the first place. And that is just to say that there would be no reason to be a minimalist.

\section{Extended-minimalism and possible extensions of English}

I have shown that minimalism cannot be formulated in terms of some one-place function (a propositional structure). I now want to consider a minimalist who is prepared to concede this point, and who attempts to meet the challenge of comprehensiveness in some other way. This response was suggested by Horwich (1998, p. 19n3) himself, as a fallback in the event that he was forced to abandon the idea that MT indicates a one-place functions.

Suppose that, for any proposition, there is some (possible) extension of English in

6 The truth operator is a monadic sentential connective, $\mathrm{T}$, which we can characterise in several ways. Semantically: the semantic value of $\mathrm{T} \varphi$ is always the same as the semantic value of $\varphi$. Proof-theoretically: the introduction rule is $\varphi \Rightarrow \mathrm{T} \varphi$; the elimination rule is $\mathrm{T} \varphi \Rightarrow \varphi$. The redundancy of the operator is obvious: reading $\mathrm{T} \varphi$ as $\varphi$ vindicates both the semantic and the proof-theoretic rules governing the operator. 
which that proposition can be expressed. This supposition is prima facie plausible, since if the proposition is expressible at all, it is presumably expressible by augmenting English with a new phrase. In that case, we could then meet the challenge of comprehensiveness by offering the following stipulation: the theory of truth is to consist of exactly the propositions which are expressed by any instance of the scheme MT in any possible extension of English (as understood in that extension of English).

Of course, MT still contains angled brackets. In order to make sense of these, we shall have to read MT as something like:

$\mathrm{MT}_{E}$. the proposition expressed by ' $p$ ' (in this extension of English) is true iff $p$

For obvious reasons, I shall call this proposal extended-minimalism.

Extended-minimalism represents a significant departure from minimalism. Indeed, I shall argue that it is such a departure from minimalism, that it is scarcely distinct from alternative deflationary approaches to truth. I shall then argue that the extended-minimalist has not succeeded in meeting the challenge of comprehensiveness.

Converging with disquotationalism. Among deflationary theories of truth, the closest contemporary rival to minimalism is probably disquotationalism. Like minimalism, disquotationalism begins with a scheme, which might be something like:

DT. ' $p$ ' (in this language) is true iff $p$

The disquotationalist then claims that this scheme exhausts the concept of truth.

As it stands, disquotationalism falls far short of the challenge of comprehensiveness. After all, DT only provides truth conditions for sentences in this language, and tells us nothing about the truth of sentences from other language. To avoid this point, the disquotationalist is likely to supplement her theory of truth with a theory of translation. She can then apply the truth-predicate to sentences of other languages, by coupling DT with the clause:

for any language $L$, and any sentence $x: x$ (in $L$ ) is true iff there is some sentence $y$ such that $y$ (in this language) is the translation of $x$ (in $L$ ) and $y$ (in this language) is true.

This goes some distance towards meeting the challenge of comprehensiveness. Unfortunately, it does not go all the way: we are left in the dark concerning any sentences (in other languages) which cannot be translated into a sentence of this language. (Compare the minimalist's difficulty, concerning propositions which are inexpressible in this language.)

Some disquotationalists are prepared simply to bite the bullet on this problem, and deny that truth extended so far. Field (1994, p. 250) was one such disquotationalist; but he soon came to accept that this violates our ordinary understanding of truth. So, in order to meet the challenge of comprehensiveness, he suggested that we should not regard DT as an 
axiom scheme for some fixed language (this one). Rather, we must be committed to extending $[\mathrm{DT}]$ as we expand the language' for any 'potential expansion' of our language (Field 2001, pp. 147-8). Call the resulting position quasi-disquotationalism.

As minimalism has become extended-minimalism, and disquotationalism has become quasi-disquotationalism, the two rival theories of truth have all but converged. The first difference was overcome when the minimalist glossed intensional ascent in terms of semantic ascent. At this point, it shared with disquotationalism the idea that linguistic expressions (of this language) are central to the analysis of truth. A difference still remained, since minimalists attribute truth to propositions expressed by sentences, rather than to the sentences themselves. But the significance of this difference has dwindled, now that quasidisquotationalists have appealed to a theory of translation which extends the truth condition of a sentence of this language to all equi-translatable sentences of other languages. Furthermore, the extended-minimalist and the quasi-disquotationalist have offered parallel responses to the challenge of comprehensiveness. In particular: both have recognised that they cannot treat their initial 'scheme' - be it $\mathrm{MT}_{E}$ or $\mathrm{DT}$ - as an axiom scheme in any standard sense. The extended-minimalist contends that we are committed to any proposition expressed by any instance of $\mathrm{MT}_{E}$ in any 'possible extension' of English, and the quasidisquotationalist contends that we are committed to any instance of DT in anything we regard as a 'potential expansion' of our language. What differences remain represent only slight variations within a camp, rather than distinct positions on the philosophical landscape. ${ }^{7}$

The point of all of this is simple. Minimalism purported to be a distinct rival to other deflationary theories of truth. However, once we have given up on the idea that the theory of truth can be formulated in terms of propositional structure, we have given up on much that was distinctive about minimalism. Of course, this is not (yet) to say that the ensuing position is wrong. It is merely to note that there are fewer varieties of deflationism than the literature suggests.

The challenge of comprehensiveness again. I now want to criticise extended-minimalism directly, by focussing again on the challenge of comprehensiveness. To frame the problem, I need to clarify what, exactly, extended-minimalism amounts to.

In invoking possible extensions of English, the extended-minimalist is not merely maintaining that $\mathrm{MT}_{E}$ is true on every (possible) assignment of meaning to ' $p$ '. For one thing, that proposal would not yet provide us with any guidance on how to deal with the left-most occurrence of ' $p$ ' in $\mathrm{MT}_{E}$, where it occurs in the context 'the proposition expressed by " $p$ " (in this extension of English)'. For another, the extended-minimalist would have invoked the notion of truth in the course of outlining the theory which was supposed itself to exhaust the concept of truth. In so doing, she would surely have undercut her claim to be any kind of

7 cf. Field (2001, p.148). On one particular point of variation: Buchanan (2003, pp. 64-5) is rightly critical of Field's suggestion that whether an utterance (in some other language) is true depends upon how I regard that utterance. 
minimalist. ${ }^{8}$

Instead, the extended-minimalist's proposal must be something more like the following: Any possible usage of an $\mathrm{MT}_{E}$-instance (where that usage counts as an extension of English) expresses a proposition which figures in the minimal theory of truth.

My concern with this proposal is relatively straightforward: it does not amount to a systematic specification of a comprehensive theory of truth.

To make this point, it will help if I first draw a couple of parallels. In $\$ I V$, I imagined a minimalist who suggested that her theory of truth consisted of all of the correct instances of $\mathrm{MT}_{2}$, but refused to say anything more about what correctness amount to. I complained that, if she followed this course, she would not have systematically specified a comprehensive theory of truth. In particular, she would not have provided us with any kind of tractable test, which applied to each and every proposition, and determined whether or not it belonged within her theory of truth. Similarly: when a correspondence theorist tells us that truth consists in correspondence, and (inevitably) does not then go on to articulate this notion in any detail, she cannot thereby claim to have systematically specified a comprehensive theory of truth (I shall return to this point in $\ I X$ ). Again: she has not provided us with a tractable test to determine which propositions belong within her theory of truth.

My concern here is exactly the same. The extended-minimalist attempts to specify a theory of truth by quantifying over all possible linguistic usage. So, until she has provided a theory which details all possible linguistic usage, by all possible creatures, in all possible contexts, she has not systematically specified a comprehensive theory of truth. Until she has provided such a theory of all possible usage, the extended-minimalist has not given any kind of tractable test, which applies to each and every proposition, and sorts them into those which belong within her theory of truth and those which do not. However, it is simply fantastical to suppose that we actually have such a theory of all possible usage; or, indeed, that we ever could have one.

8 Horwich (1998, pp. 25-7) makes similar points himself, in a slightly different context. 


\section{The importance of comprehensiveness}

We keep bumping up against the challenge of systematically specifying a comprehensive theory of truth. As a final move, then, it is worth asking why a deflationist should feel compelled to take on this challenge. In particular, the analogy with the correspondence theorist in the previous section might motivate a deflationist to make the following speech: ${ }^{9}$

The correspondence theorist claims that the essence of truth is that it consists in correspondence. She does not thereby claim to have systematically specified a comprehensive theory of truth. And no one faults her for this, particularly. Rather, they fault her (if at all) on the grounds that the idea of 'correspondence' is obscure and useless.

In a similar spirit: I maintain that truth has no essence, so that truth does not consist in correspondence, or in anything else. I add, by way of positive account, that the truth predicate is a device whose utility is exhausted by its role in allowing us to denominalise content.

Now: why, in saying this, should I be obliged to provide a comprehensive theory of truth, when you do not impose the same demand on the correspondence theorist?

In reply to this speech, it would be inadequate simply to repeat the argument in favour of comprehensiveness that I presented in SII. There, I pointed out only that a noncomprehensive theory would omit some truth condition, and so omit something about truth. Our imagined deflationist has freely conceded this point, and she does not much care, for her point is just that truth has no essence.

There is, though, an important difference between the correspondence theorist and our imagined deflationist. Our deflationist seeks to convince us that there is very little to say about truth in general. Establishing this negative claim is rather hard. Indeed, the literature on deflationism is littered with accusations from anti-deflationary parties that there are some important general facts which (essentially) involve truth. The now-classic deflationist strategy for dealing with such objections - employed by both Horwich (e.g. 1998, pp. 2025, 46; 2001, pp. 160-1; 2009a, pp. 48-50) and Field (1994, pp. 264-5) - is to attempt to 'derive' the general fact, from the deflationary theory of truth, together with some separate theory which does not mention truth.

As an instance of this general strategy, we can consider Horwich's attempted 'derivation' of the (supposed) general fact that it is desirable to believe all and only truths (2009, p. 49). We start with a truth-free normative theory, consisting of all 'norms of the form':

9 This speech was inspired by some probing questions from Daniel Nolan (though I do not want to put the speech in his mouth) and also by a remark by Armour-Garb (2012, p. 275). 
(a) It is desirable that (one believe $\langle p\rangle$ iff $p$ )

Next, given all propositions 'of the form' illustrated by MT, we arrive at all 'norms of the form':

(b) It is desirable that (one believe $\langle p\rangle$ iff $\langle p\rangle$ is true)

And hence, by some supposed mechanism for generalisation, at:

(c) $\forall x$ (It is desirable that (one believe $x$ iff $x$ is true))

In explaining the strategy, I have followed Horwich in using the locution 'of the form'; however, the considerations of this paper show that we should be very suspicious of the phrase. That said, the shape of the general strategy is clear enough. Moreover, we can see how it would be adopted to fit a raft of alternative deflationary theories of truth.

The general strategy faces several very serious obstacles in-principle (raised by Anil Gupta (1993), Panu Raatikanen (2005), and Marian David (2007; 2008), among others). Even waiving these, however, one thing is absolutely clear: the general strategy has no hope of succeeding, unless the deflationist has access to a comprehensive theory of truth. If, for example, the deflationist could only specify the truth conditions for those propositions which are expressible in this language, then she will (at best) be able to explain the value of the truths expressible in this language, and not the value of truth per se. The situation is even worse if the deflationist has utterly eschewed the aim of systematically specifying truth conditions; for in that case, we cannot even begin the purported derivation.

In short: if the deflationist wants to be able to convince us that she has not omitted any important facts about truth, then she will need to provide us with a theory of truth that addresses the challenge of comprehensiveness. And that is exactly what she cannot do.

\section{Demotivating minimalism}

In this paper, I have argued against minimalism. But knowing that minimalism is wrong might not remove its temptations. With this in mind, I shall close this paper by rephrasing its main argument slightly, with the aim of undermining one of the main temptations towards minimalism.

In the first paragraph of the preface to the first edition of his book Truth, Horwich sets out his minimalist programme:

Perhaps the only points about truth on which most people could agree are, first, that each proposition specifies its own condition for being true (e.g. the proposition that snow is white is true if and only if snow is white), and, second, that 
the underlying nature of truth is a mystery. The general thrust of this book is to turn one of these sentiments against the other. I want to show that truth is entirely captured by the initial triviality, so that nothing could be more mundane and less puzzling than the concept of truth. (Horwich 1998, p. ix; Horwich's emphasis)

I want to focus on the first claim, 'that each proposition specifies its own condition for being true'. This is not a throwaway remark from Horwich; elsewhere he says:

the central principle governing our overall deployment of the truth predicate is, very roughly, that each statement articulates the conditions that are necessary and sufficient for its own truth. (Horwich 2009e, p. 15)

[The minimalist] strategy focuses on the way that every statement trivially specifies its own condition for being true. (Horwich 2009f, p. 3)

Suppose Horwich were right about all this. Then it would then be a trivial matter to provide truth conditions for each and every proposition: just let each proposition specify its own truth condition. But, as the historical record shows, it is very hard to say anything more about truth, beyond these (trivial) specifications of truth conditions. The reasonable thought now arises, that there genuinely is nothing more to say. And so we have arrived at minimalism.

The preceding line of thought is very seductive. However, the considerations of this paper show that it is flawed at the outset.

Certainly it is tempting to say that the proposition that snow is white specifies its own truth condition. After all, when we pick it out as the proposition that snow is white, its truth condition is immediately obvious. However, we are not obliged to pick out propositions by using the locution 'the proposition that...'. We might, for example, pick out a proposition as Tarski's favourite proposition. Equally, we might pick out a proposition using some primitive name, such as ' $q$ '. There is surely no temptation to say that Tarski's favourite proposition, or $q$, specifies its own truth condition. And this, even though Tarski's favourite proposition, and $q$, both just happen to be the proposition that snow is white.

This indicates that propositions themselves do not specify their own truth conditions. Rather, a proposition's truth condition is specified (in this way) precisely when the proposition is picked out using the locution 'the proposition that...' (or, equivalently, using angled brackets).

Of course, the use of this locution amounts to something like our canonical method for picking out propositions. And the very centrality of this locution explains why we are tempted to say (mistakenly) that propositions specify their own truth conditions. Moreover, if we could pick out every proposition by using a phrase of the form 'the proposition that...', then it would simply be a harmless oversimplification to maintain that a proposition specifies its own truth conditions. Whilst we could not rely on propositions to specify their own truth 
conditions, we could rely on their canonical presentations to do the job for us.

At this point, however, the considerations of this paper kick in. This language does not enable us to pick out every proposition using the locution 'the proposition that...'. Moreover, our understanding of that locution invokes semantic ascent in this language. In short: the claim that a proposition specifies its own truth condition ignores the role played by semantic ascent within this language. And this is no longer a harmless oversimplification. It conceals the fact that minimalism cannot address the challenge of comprehensiveness. ${ }^{10}$

\section{References}

Anscombe, G. E. M. 1957: 'Report on Analysis “Problem” no. 10'. Analysis, 17(3), pp. 49- 52.

Armour-Garb, Bradley 2012: 'Deflationism (About Theories of Truth)'. Philosophy Compass, 7(4), pp. 267-77.

Buchanan, Ray 2003: 'Are Truth and Reference Quasi-Disquotational?' Philosophical Studies, 113, pp. 43-75.

David, Marian 2007: 'Review of Horwich's From a Deflationary Point of View'. Mind, 116(426), pp. 427-34.

- 2008: 'Quine's Ladder: Two and a Half Pages from the Philosophy of Logic'. Midwest Studies in Philosophy, 32, pp. 274-312.

Davidson, Donald 1990: 'The Structure and Content of Truth'. The Journal of Philosophy, 87(6), pp. 279-328.

- 1996: 'The Folly of Trying to Define Truth'. The Journal of Philosophy, 93(6), pp. 263-78.

Field, Hartry 1992: 'Review of Horwich's Truth'. Philosophy of Science, 59(2), pp. 321-30.

- 1994: 'Deflationist Views of Meaning and Content'. Mind, 103(411), pp. 249-85.

- 2001: 'Postscript to Field 1994'. In his Truth and the Absence of Fact. New York: Oxford University Press, pp. 141-56.

Gupta, Anil 1993: 'A Critique of Deflationism'. Philosophical Topics, 21(2), pp. 57-81.

Horwich, Paul 1998: Truth. Second edition. Oxford: Oxford University Press.

- 2001: 'A Defense of Minimalism'. Synthese, 126, pp. 149-65.

- 2009a: 'A Defense of Minimalism'. In his 2009d, pp. 35-56.

- 2009b: 'A Minimalist Critique of Tarski'. In his 2009d, pp. 79-98.

- 2009c: 'The Value of Truth'. In his 2009d, pp. 57-78.

- 2009d: Truth-Meaning-Reality. Oxford: Oxford University Press.

- 2009e: 'Varieties of Deflationism'. In his 2009d, pp. 13-34.

- 2009f: 'What is Truth?'. In his 2009d, pp.1-12.

Potter, Michael 2000: Reason's Nearest Kin: Philosophy of Mathematics from Kant to Carnap. Oxford:

Oxford University Press.

10 Particular thanks to George Bealer, James Cargile, Branden Fitelson, Richard Holton, Peter van Inwagen, Mark Jago, Daniel Nolan, Fredrik Nyseth, Michael Potter, Ted Sider, Rob Trueman, and Meg Wallace. Further thanks to audiences at the Aristotelian Society, Cambridge, Helsinki, Kent, Nottingham, Stirling, and Tucson. 
Raatikainen, Panu 2005: 'On Horwich's Way Out'. Analysis, 65(3), pp. 175-7.

Reach, Karl 1938: 'The Name Relation and the Logical Antinomies'. Journal of Symbolic Logic, 3(3), pp. 97-111.

Read, Stephen 1997: 'Quotation and Reach's Puzzle'. Acta Analytica, 19, pp. 9-20.

Russell, Bertrand 1905: 'On Denoting'. Mind, 14(56), pp. 479-93. 